Jan Behrends, Ifor D. W. Samuel, Alexander Schnegg and David J. Keeble: Persistent spin coherence and bipolarons. Nature Nanotechnology 8:884-885, 2013. http://dx.doi.org/10.1038/nnano.2013.269. (†)

\title{
Organic Semiconductors: persistent spin coherence and bipolarons
}

In their recent commentary Boehme and Lupton ${ }^{1}(\mathrm{BL})$ discuss the current challenges for organic spintronics, and correctly highlight the importance of spectroscopic methods that directly detect the participating spin species. We have previously observed room temperature coherent electron spin Rabi oscillations persisting beyond $500 \mathrm{~ns}$ by pulsed electrically detected magnetic resonance ( $\mathrm{pEDMR}$ ) from a conventional bulk heterojunction organic solar cell $^{2}$, comprising a blend of $20 \%$ conjugated polymer (MEH-PPV) and $80 \%$ PCBM $\left(\mathrm{C}_{60}\right)$. We also observed spin-locking with high microwave magnetic field amplitudes and the results proved unambiguously that the two spin species responsible were $S=1 / 2$. This observation eliminated models for the spin dependent transport, which provide sensitivity to magnetic fields, such as quenching of triplet excitons by polarons or mutual annihilation of two triplet excitons. Considering both the spectroscopic evidence and the device physics we concluded that the spin dependent transport mechanism involved hopping via doubly occupied polymer segments, i.e. bipolaron states.

BL discuss the importance of distinguishing between spin dependent transport resulting from bipolaron mediated hopping and polaron pair recombination $\left(P^{+} P^{-}\right)$and in so doing question our conclusions. It is indeed worthwhile to review the evidence supporting these models. The overlap of pEDMR spectra complicates the interpretation, so careful consideration of the device structures and operating conditions used for these experiments is necessary. It is important to note that our measurements were made on blends, whereas BL studied neat films.

Our pEDMR measurements on blend devices observed a spectrum at the $g$-value $(2.0028(3))$ characteristic of polarons in MEH-PPV enabling us to conclude that these were the responsible spin species. Two contributions to the spectrum could be resolved, both with the

$(\dagger)$ Disclaimer: The publisher's version overrides in case of any discrepancy. 
same $g$-value but with linewidths of $0.6(1) \mathrm{mT}$ and $1.5(1) \mathrm{mT}$, respectively ${ }^{2}$. The pEDMR spectrum from a pure MEH-PPV device observed by Boehme, Lupton, and co-workers ${ }^{3,4}$, also shows a spectrum at $g=2.003$ comprising two components, but with linewidths of approximately $1.3 \mathrm{mT}$ and $3 \mathrm{mT}$. BL show a new room temperature Rabi oscillation spectra from a similar pure MEH-PPV device (Figure 1b of BL) and compare these results with those from our blend device (Figure 1a). The only unambiguous conclusion that follows from the similarity shown is that in both types of device two $S=1 / 2$ entities are responsible for the spin dependent transport. The onset of spin locking depends on the degree of spectral overlap for the two contributing species, and the linewidth of the narrower spectral component. The apparent similarity of the onset microwave $\mathrm{B}_{1}$ value (Figure 1a, b) is puzzling, given the differences in linewidths for the two pEDMR spectra from the blend and pure devices.

BL proceed to show a new $\mathrm{pEDMR}$ spectrum for a pure PCBM film at room temperature (Figure 1c). It comprises two components, one narrow the other broad, $\sim 3.5 \mathrm{mT}$, centered at a $g$-value of $\sim 2.002$, and is similar to a previous result from a pure $\mathrm{C}_{60}$ film which exhibited coincident components with linewidths of $\sim 0.3 \mathrm{mT}$ and $\sim 3 \mathrm{mT}^{5}$. The EPR spectrum of the radical anion, $P^{-}$, on PCBM has a $g$-value of 1.9995 and a linewidth of $\sim 0.3 \mathrm{mT}$ at $100 \mathrm{~K}^{6,7}$. In addition, it has been shown that the signature of the PCBM anion localized at the heterojunction interface is similar ${ }^{7}$. However, the identity of the spin partners responsible for the observed pEDMR spectra from fullerene thin films has yet to be established. It is also important to note that no spin locking was observed in the study of pure $\mathrm{C}_{60}$ films ${ }^{5}$, indicating this signal is different in nature from that we observed in the blend device.

BL propose that our pEDMR spectrum from the blend comprises a superposition of two independent $P^{+} P^{-}$recombination processes, one exclusive to MEH-PPV, the other on PCBM. We disagree, there is no spectroscopic evidence for the involvement of spin entities within the PCBM component of the blend. The spectrum we observed showed a different $g$-value 
and is noticeable narrower than that shown in Figure 1c. The absence of spin locking in pEDMR of pure fullerene films provides evidence against a contribution from a recombination process exclusive to the PCBM component. The lack of involvement of spin entities from the PCBM was perhaps surprising, since an obvious spin dependent process would be a $P^{+} P^{-}$recombination processes occurring at the heterojunction interfaces between MEH-PPV $P^{+}$and PCBM $P^{-}$.

We agree that $P^{+} P^{-}$recombination on MEH-PPV is responsible for the spin dependent contribution, comprising on the order of 1 in $10^{4}$ of the transport current ${ }^{4}$, in the pure MEHPPV devices. This is supported by evidence that the $g$-value for $P^{-}$is similar to that for the positive polaron ${ }^{8}$. However, the situation is different in the blend devices; while the pEDMR spectra again show two contributions at the polaron $g$-value the linewidths are different from those in the pure devices, and importantly the device physics is markedly altered by the inclusion of PCBM. The presence of a high density of heterojunction interfaces with PCBM, at which the band offsets present a $\sim 1 \mathrm{eV}$ energy gain for $P^{-}$transfer to PCBM, means that the probability of negative polarons being present in the conjugated polymer component is negligible. Further, the pEDMR on the blend devices was performed with low bias $(U=1 \mathrm{~V})$ inhibiting electron injection to MEH-PPV. By contrast, the pEDMR spectra from the pure MEH-PPV devices used medium to high applied bias values $(U \sim 4-15 \mathrm{~V})$. Under these conditions the electrical injection of $P^{+}$and $P^{-}$is possible so $P^{+} P^{-}$recombination is highly plausible.

In consequence, we maintain our assertion that the data suggest that the most probable mechanism explaining the pEDMR in the MEH-PPV:PCBM blend device is the percolation transport of $P^{+}$mediated by transient spin dependent bipolaron formation from weakly coupled $P^{+} P^{+}$precursor states. BL claim the bipolaron model describes an energetically unfavorable "new particle". The suggestion that bipolarons in conjugated polymers are "new" 
is wrong: they were proposed more than 30 years ago ${ }^{9}$ and are supported by experimental evidence including Ref. 10.

The key importance of magnetic field sensitive transport in organic semiconductors requires that we fully explore and test the models for spin dependent transport. This requires careful examination of spectroscopic evidence and the device physics, and should result in a cohesive and predictive framework. The key result of our paper, namely long-lived spin coherence in a polymer:fullerene blend at room temperature is unchallenged and together with work on the neat materials suggest the possibility of coherent spin manipulation on the microsecond timescale at room temperature ${ }^{2,4,5}$. The understanding and application of such spin phenomena is a promising future direction for organic semiconductors.

Jan Behrends ${ }^{1}$, Ifor D. W. Samuel ${ }^{2}$, Alexander Schnegg ${ }^{3}$, and David J. Keeble ${ }^{4}$

${ }^{1}$ Fachbereich Physik, Freie Universität Berlin, Arnimallee 14, D-14195 Berlin, Germany

${ }^{2}$ Organic Semiconductor Centre, SUPA, School of Physics and Astronomy, SUPA, University of St. Andrews, St. Andrews KY16 9SS, United Kingdom

${ }^{3}$ Helmholtz-Zentrum Berlin für Materialien und Energie, Kekuléstraße 5, D-12489 Berlin, Germany

${ }^{4}$ SUPA, Division of Physics, University of Dundee, Dundee DD1 4HN, United Kingdom 
1 Boehme, C. \& Lupton, J. M. Challenges for organic spintronics. Nature Nanotech. 8, 612-615, (2013).

2 Behrends, J. et al. Bipolaron Formation in Organic Solar Cells Observed by Pulsed Electrically Detected Magnetic Resonance. Phys. Rev. Lett. 105, 176601, (2010).

3 McCamey, D. R. et al. Hyperfine-Field-Mediated Spin Beating in Electrostatically Bound Charge Carrier Pairs. Phys. Rev. Lett. 104, 017601, (2010).

4 Baker, W. J., Keevers, T. L., Lupton, J. M., McCamey, D. R. \& Boehme, C. Slow Hopping and Spin Dephasing of Coulombically Bound Polaron Pairs in an Organic Semiconductor at Room Temperature. Phys. Rev. Lett. 108, (2012).

5 Harneit, W. et al. Room temperature electrical detection of spin coherence in $\mathrm{C}_{60}$. Phys. Rev. Lett. 98, 216601, (2007); ibid 100, 199904, (2008).

6 De Ceuster, J., Goovaerts, E., Bouwen, A., Hummelen, J. C. \& Dyakonov, V. Highfrequency $(95 \mathrm{GHz})$ electron paramagnetic resonance study of the photoinduced charge transfer in conjugated polymer-fullerene composites. Phys. Rev. B 64, 195206, (2001).

7 Behrends, J. et al. Direct detection of photoinduced charge transfer complexes in polymer fullerene blends. Phys. Rev. B 85, (2012).

8 Zezin, A. A., Feldman, V. I., Warman, J. M., Wildeman, J. \& Hadziioannou, G. EPR study of positive holes on phenylene vinylene chains: from dimer to polymer. Chem. Phys. Lett. 389, 108-112, (2004).

9 Brazovskii, S. A. \& Kirova, N. N. Excitons, polarons, and bipolarons in conducting polymers. JETP Lett. 33, 4-8, (1981).

10 Ziemelis, K. E. et al. Optical spectroscopy of field-induced charge in poly(3-hexyl thienylene) metal-insulator-semiconductor structures - evidence for polarons. Phys. Rev. Lett. 66, 2231-2234, (1991). 


\section{Competing financial interests}

The authors declare no competing financial interests. 